\title{
A study on the Correlation between BMI \& Carotid Atherosclerosis and Bone Metabolism in Perimenopausal Women
}

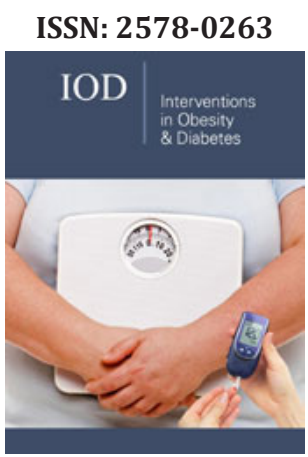

*Corresponding author: CuiFeng Zhu, Department Director of Clinical Nutrition, Chief Physician, Master Tutor of Shenzhen Hospital of Southern Medical University, Post Doctor Fellows of Department of Endocrinology of University of Hong Kong \& The State Key laboratory of Pharmaceutical Biotechnology, China

Submission: 每韭 March 11, 2020

Published: 想April 23, 2020

Volume 4 - Issue 1

How to cite this article: Jianguo Liu, Liehui Xiao, Yong Pan, Cuifeng Zhu, et al. A study on the Correlation between BMI \& Carotid Atherosclerosis and Bone Metabolism in Perimenopausal Women. Interventions Obes Diabetes 4(1). IOD.000577. 2020 DOI: 10.31031/IOD.2020.04.000577

Copyright@ Cuifeng Zhu. This article is distributed under the terms of the Creative Commons Attribution 4.0 International License, which permits unrestricted use and redistribution provided that the original author and source are credited.

\author{
Jianguo Liư ${ }^{1}$, Liehui Xiao ${ }^{1}$, Yong Pan ${ }^{2,3}$, Xiuping Lin ${ }^{1}$, Yuan Zhang ${ }^{1}$, JinChuan \\ Cai $^{1}$, Yan Liu ${ }^{1}$, Zhentian Zhang ${ }^{1}$, Muxiu Yang ${ }^{1}$, Leijun Zhang ${ }^{1}$, Aimin $\mathrm{Xu}^{2,3}$ and \\ Cuifeng Zhu ${ }^{1,2,3 *}$ \\ ${ }^{1}$ Shenzhen Hospital of Southern Medical University, China \\ ${ }^{2}$ Department of Medicine, Li Ka Shing Faculty of Medicine, China \\ ${ }^{3}$ The State Key laboratory of Pharmaceutical Biotechnology, China
}

\begin{abstract}
Objective: To investigate the correlation between BMI \& carotid atherosclerosis and bone metabolism in perimenopausal women.

Methods: Collected in March 2017 to July 2019 in shenzhen, southern medical university hospital health management center to accept physical examination of 89 patients with perimenopausal women had investigated and analyzed the clinical data were retrospectively according to whether the carotid atherosclerosis was divided into carotid sclerosis group and normal group, compared two groups of patients with clinical data, BMI, bone metabolism index, analysis of carotid atherosclerosis and bone metabolic index level of correlation.
\end{abstract}

Results: Among the 89 perimenopausal women, 25 had carotid atherosclerosis (the prevalence was $28.09 \%$ ). The content of hypertension component ratio, diabetes component ratio, body mass index (BMI), carotid intima-media thickness (IMT), TG ,LDLc and parathyroid hormone (PTH) in the carotid atherosclerosis group was significantly higher than that in the normal group $(\mathrm{P}<0.05$ or $\mathrm{P}<0.01)$. However, the levels of high-density lipoprotein cholesterol (HDL-c), 25-hydroxyvitamin $\mathrm{D}_{3}\left[25(\mathrm{OH}) \mathrm{D}_{3}\right]$, serum type I collagen cross-linked c-terminal peptide (CTX) and osteocalcin were all lower than the normal group $(\mathrm{P}<0.05$ or $\mathrm{P}<0.01)$. Spearman correlation analysis showed that BMI, TG and PTH levels were positively correlated with IMT $(\mathrm{r}=5.513,4.433,5.264, \mathrm{P}<0.05)$, while ldl-c, 25(OH) $\mathrm{D}_{3}$, CTX and osteocalcin levels were negatively correlated with IMT $(r=-4.402,-4.508,-4.465,-4.406, \mathrm{P}<0.05)$. Multivariate Logistic regression analysis showed that hypertension,diabetes, Overweight or obese, dyslipidemia, and osteocalcin declining were risk factors for cervical atherosclerosis in perimenopausal women $(\mathrm{P}<0.05)$.

Conclusion: Decreased osteocalcin level, increased TG and BMI, hypertension and diabetes are therisk factors for carotid atherosclerosis in perimenopausal women.

Keywords: Perimenopausal women; BMI; Carotid atherosclerosis; Bone metabolism; Osteocalcin

\section{Introduction}

Perimenopause mainly refers to the stage when women transition from reproductive age to old age, with decreased estrogen and dysfunction of vegetative nerves, leading to obesity, hypertension, coronary heart disease, dizziness, insomnia, emotional instability and other symptoms in women at this stage [1]. The decrease of estrogen content in perimenopausal women reduces the regulation of body fat mass, blood lipid and the protection of blood vessels, leading to an increased risk of vascular diseases [2]. As one of the vascular diseases, atherosclerosis is a systemic and diffuse disease, which causes the involvement of large and medium-sized arteries in the circulatory system and changes in the structure and function of various important organs. Neck is a frequently involved part of arteriosclerosis, and arteriosclerotic plaque in the neck is one of the characteristics of arteriosclerosis. Estrogen is an important hormone to maintain female bone mineral content,which is conducive to bone formation. In perimenopausal women, the function of warm nest gradually declines, which directly causes the decline of estrogen, the imbalance of bone reconstruction, and the bone absorption rate is higher than the bone formation rate, leading to the occurrence of osteoporosis [3]. In recent years, the drop in estrogen caused the change of bone metabolism index correlation with disease of lipid metabolism and heart head blood-vessel is becoming 
a hot spot of perimenopausal women's health study, the paper discusses perimenopausal women BMI \& carotid atherosclerosis and bone metabolic index level of correlation, expect for perimenopausal women provides the reference for the tertiary prevention of cardiovascular disease and train of thought, its research content reported as follows.

\section{Data and Methods}

General information: the clinical data of 89 perimenopausal women who underwent physical examination in the health management center of Shenzhen hospital of Southern Medical University from March 2017 to July 2019 were retrospectively investigated. Inclusion criteria for perimenopausal women: age $\geq 45$ years; The duration of menopause is no more than 12 months, during which menstruation may be irregular and hormone level may change. he has not received hormone therapy recently. Exclusion criteria: patients with severe heart, liver, kidney or infectious diseases; Patients with malignant tumors; Taking calcium supplements, hormones or drugs that affect bone metabolism for a long time.

\section{Method}

The patients' age, menstrual history, personal history and previous history were recorded, and BMI was calculated. Fasting venous blood was extracted in the early morning, and the levels of total cholesterol (TC), triglyceride (TG), low-density lipoprotein cholesterol (LDL-c) and high-density lipoprotein cholesterol (HDL-c) were measured by automatic biochemical analyzer. Using automatic electrochemical luminescence analyzer to detect bone metabolism index levels, including parathyroid hormone (PTH), 25-hydroxyl vitamin $\mathrm{D}_{3}\left[25(\mathrm{OH})_{2} \mathrm{D}_{3}\right]$, serum collagen type the c-terminal peptide (CTX), crosslinking type tropocollagen amino terminal propeptide (PINP) and osteocalcin. In addition, color doppler ultrasound was used to detect the carotid intima media thickness and the occurrence of atherosclerotic plaque in perimenopausal women. According to the standards in the "guidelines for vascular ultrasound examination" formulated by the sonographer branch of the Chinese medical doctors association [4], carotid intima media thickness (IMT) $\geq 1 \mathrm{~mm}$ indicated the presence of intima thickening, and the presence of local $\geq 1.5 \mathrm{~mm}$ was considered as plaque.

\section{Statistical method}

SPSS25.0 statistical software was used, and the measurement data were expressed as mean \pm standard deviation $( \pm s)$. Independent sample $t$ test was used for the difference analysis between normally distributed data groups, while mann-whitney $\mathrm{U}$ test was used for the difference analysis between non-normally distributed data groups. The counting data were expressed as the number of cases (composition ratio), and the difference between groups was analyzed by 2 test or Fisher test. Spearman was used to analyze the correlation between indicators. Logistics using multiple factors regression, analysis of perimenopausal women carotid atherosclerosis and bone metabolic indices such as index of correlation, and analyzes the possible associated risk factors, inspection level $a=0.05$.

\section{Result}

Compare two groups of patients with basic data Twenty-five of the 89-perimenopausal women developed carotid atherosclerosis, with a prevalence rate of $28.09 \%$. The comparison and analysis of the basic data of the two groups of patients showed that there were no statistically significant differences in age, composition ratio of alcohol consumption, composition ratio of smoking, LDLc level, TC level and PINP content between the twogroups of perimenopausal women $(\mathrm{P}>0.05)$. BMI, IMT, composition ratio of hypertension, composition ratio of diabetes mellitus, TG level and PTH content of perimenopausal women in the carotid atherosclerosis group were higher than those in the normal group $(\mathrm{P}<0.05$ or $\mathrm{P}<0.01)$, while HDLc, $25(\mathrm{OH}) \mathrm{D}_{3}$, CTX and osteocalcin content were lower than those in the normal group $(\mathrm{P}<0.05$ or $\mathrm{P}<0.01)$, as shown in Table 1 \& Figure 1.

Table 1: Comparison of basic data between the two groups of perimenopausal women. The independent sample $t$ test was used, and the mann-whitney $U$ test was used for the difference analysis and comparison between non-normal distribution data sets. The counting data were expressed as the number of cases (composition ratio), and the difference between groups was analyzed by 2 test or Fisher test. $\star \star$ means two groups of comparison $\mathrm{P}<0.01$, $\star$ means two groups of comparison $\mathrm{P}<0.05$.

\begin{tabular}{|c|c|c|c|c|}
\hline Factor & $\begin{array}{l}\text { Carotid Atherosclerosis Plaque } \\
\text { Positive Group }(n=25)\end{array}$ & $\begin{array}{l}\text { Normal Control } \\
\text { Group }(n=64)\end{array}$ & $\chi^{2} / \mathrm{t}$ & $\mathbf{P}$ \\
\hline Age(year) & $49.36 \pm 4.65$ & $50.27 \pm 4.73$ & 0.82 & 0.415 \\
\hline $\operatorname{BMI}\left(\mathrm{kg} / \mathrm{m}^{2}\right)$ & $25.68 \pm 2.06$ & $24.21 \pm 2.13$ & 2.952 & $0.004 \star \star$ \\
\hline IMT(mm) & $1.37 \pm 0.13$ & $0.62 \pm 0.18$ & 18.962 & $0.000 \star \star$ \\
\hline Drinking & & & 0.206 & 0.65 \\
\hline Yes & 10 & 29 & & \\
\hline No & 15 & 35 & & \\
\hline Smoker & & & 0.384 & 0.535 \\
\hline
\end{tabular}




\begin{tabular}{|c|c|c|c|c|}
\hline Yes & 8 & 25 & & \\
\hline No & 17 & 39 & & \\
\hline HBP & & & 4.41 & $0.036 \star$ \\
\hline Yes & 12 & 16 & & \\
\hline No & 13 & 48 & & \\
\hline Diabetes & & & 4.357 & $0.037 \star$ \\
\hline Yes & 11 & 14 & & \\
\hline No & 14 & 50 & & \\
\hline $\mathrm{TG}(\mathrm{mmol} / \mathrm{L})$ & $1.78 \pm 0.32$ & $1.48 \pm 0.27$ & 4.468 & $0.000 \star \star$ \\
\hline $\mathrm{TC}(\mathrm{mmol} / \mathrm{L})$ & $4.76 \pm 0.56$ & $4.57 \pm 0.48$ & 1.601 & 0.113 \\
\hline LDL-C(mmol/L) & $1.78 \pm 0.26$ & $1.69 \pm 0.29$ & 1.353 & 0.18 \\
\hline HDL-C(mmol/L) & $1.28 \pm 0.30$ & $1.49 \pm 0.44$ & 2.192 & $0.031 \star$ \\
\hline PTH(ng/mL) & $30.58 \pm 3.22$ & $25.46 \pm 2.69$ & 7.628 & $0.000 \star \star$ \\
\hline $25(\mathrm{OH}) \mathrm{D}_{3}(\mathrm{pg} / \mathrm{mL})$ & $13.68 \pm 1.11$ & $33.64 \pm 2.47$ & 19.361 & $0.000 \star \star$ \\
\hline CTX(ng/mL) & $0.34 \pm 0.12$ & $0.49 \pm 0.13$ & 4.995 & $0.000 \star \star$ \\
\hline PINP(ng/mL) & $50.33 \pm 5.94$ & $52.43 \pm 5.13$ & 1.659 & 0.101 \\
\hline Osteocalcin (ng/mL) & $16.68 \pm 2.78$ & $21.08 \pm 2.69$ & 6.871 & $0.000 \star \star$ \\
\hline
\end{tabular}

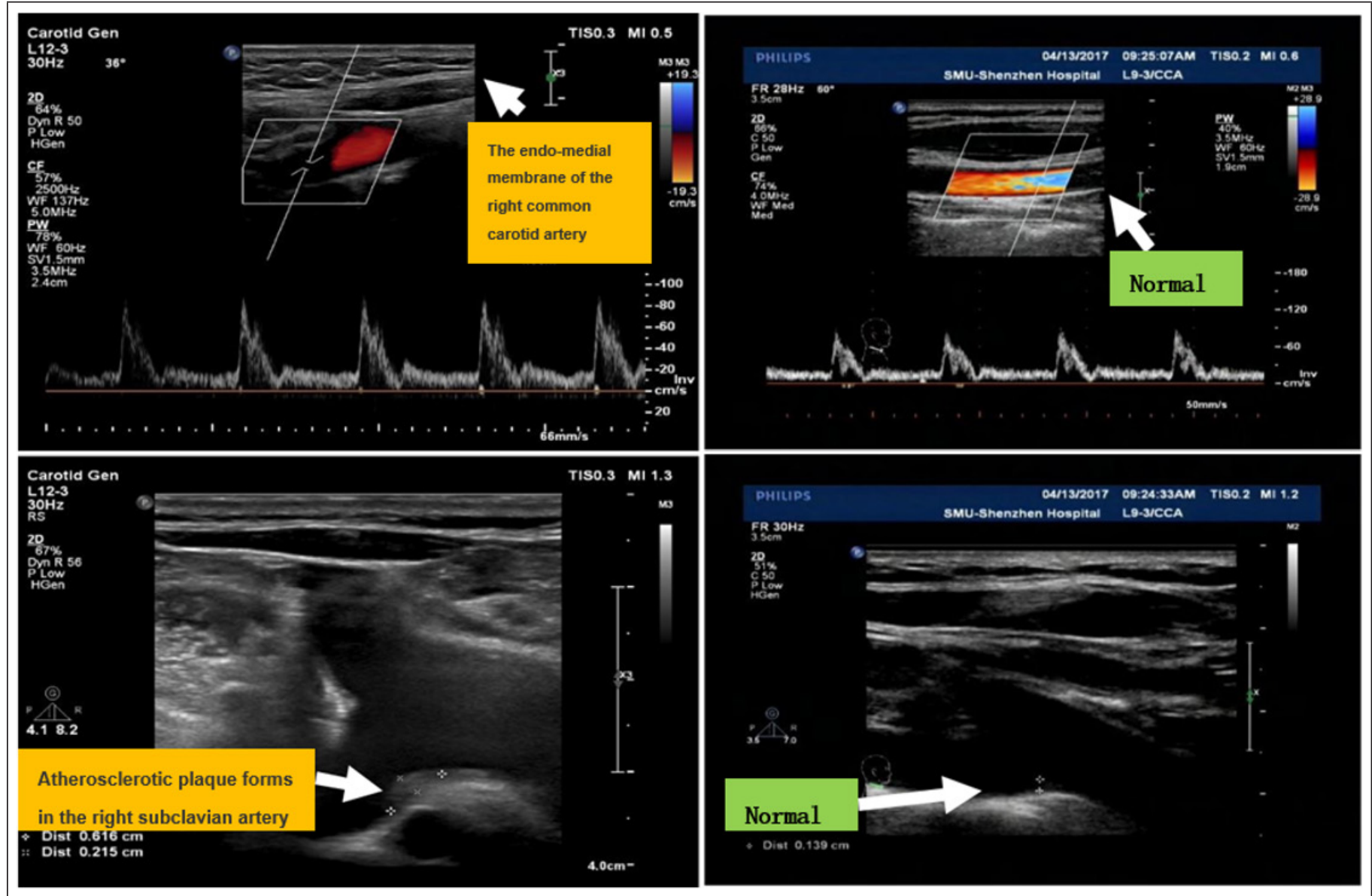

Figure 1: Ultrasonic image of atherosclerotic plaque and endo-medial membrane thick of the carotid artery compare with normal control. 
The correlation between each index level and carotid IMT

Table 2: Correlation between each indicator level and carotid IMT. Spearman analysis was used to analyze the correlation among the indicators, and $\star$ represented $\mathrm{P}<0.05$ for comparison between the two groups.

\begin{tabular}{|c|c|c|}
\hline Biomarker & $\mathbf{r}$ & $\mathbf{P}$ \\
\hline Age $($ year $)$ & 0.12 & 0.156 \\
\hline $\mathrm{BMI}\left(\mathrm{kg} / \mathrm{m}^{2}\right)$ & 5.513 & $0.016 \star$ \\
\hline $\mathrm{TG}(\mathrm{mmol} / \mathrm{L})$ & 4.433 & $0.036 \star$ \\
\hline $\mathrm{TC}(\mathrm{mmol} / \mathrm{L})$ & 0.089 & 0.267 \\
\hline $\mathrm{LDL}-\mathrm{C}(\mathrm{mmol} / \mathrm{L})$ & 0.035 & 0.304 \\
\hline $\mathrm{HDL}-\mathrm{C}(\mathrm{mmol} / \mathrm{L})$ & -4.042 & $0.043 \star$ \\
\hline $\mathrm{PTH}(\mathrm{ng} / \mathrm{mL})$ & 5.264 & $0.027 \star$ \\
\hline $25(\mathrm{OH}) \mathrm{D}_{3}(\mathrm{pg} / \mathrm{mL})$ & -4.508 & $0.042 \star$ \\
\hline $\mathrm{CTX}(\mathrm{ng} / \mathrm{mL})$ & -4.465 & $0.035 \star$ \\
\hline PINP(ng/mL) & 0.014 & 0.367 \\
\hline Osteocalcin(ng/mL) & -4.406 & $0.039 \star$ \\
\hline
\end{tabular}

Spearman correlation analysis showed positive correlation between BMI, TG and PTH, 25(OH) $\mathrm{D}_{3}, \mathrm{CTX}$, osteocalcin and IMT
$(\mathrm{P}<0.05)$, while age, TC, LDLc, and PINH levels were negatively correlated with IMT $(\mathrm{P}>0.05)$, as shown in Table 2.

\section{Multivariate Logistic regression analysis of carotid atherosclerosis in postmenopausal women}

The influencing factors with statistical significance in the basic data of the two groups of patients were used for multivariate Logistic regression analysis. Carotid atherosclerosis in menopausal women was taken as the dependent variable ("yes" was assigned to"1", "no" to "0"), BMI [" $\geq 25\left(\mathrm{~kg} / \mathrm{m}^{2}\right)$ was assigned to "1", "<25 (kg/ $\mathrm{m}^{2}$ )" was assigned to "0")], hypertension ("yes" was assigned to" 1 ", "no" was assigned to "0"), diabetes ("yes" was assigned to" 1 ", "no" was assigned to "0"),TG [" $\geq 1.7$ (mmol/L) "was assigned to" 1 ". " $<1.7$ (mmol/L)" assigned to "0"], HDLc [" $\leq 1.2$ (mmol/L)" assigned to "1", “>1.2 (mmol/L)" assigned to “0”], PTH [“ $\geq 30.0$ (ng/mL) "assigned to "1", “<30.0 (ng/mL)" assigned to "0"], 25(OH) $\mathrm{D}_{3}[$ " $\leq 13.0(\mathrm{pg} / \mathrm{mL})$ "assigned to "1", ">13.0 (pg/mL)" assigned to "0"], CTX [" $\leq 0.3$ (ng/ $\mathrm{mL}$ ) "assigned to "1", ">0.3 (ng/mL)" assigned to "0"], osteocalcin [" $\leq 16.0$ (ng/mL) "assigned to "1", "> 16.0 (ng/mL)" assigned to "0"] as independent variables. Multivariate Logistic regression analysis showed that BMI $\left(\geq 25\left(\mathrm{~kg} / \mathrm{m}^{2}\right)\right.$, hypertension, diabetes mellitus, TG ( $\geq 1.7 \mathrm{mmol} / \mathrm{L})$, and osteocalcin $(\leq 16.0 \mathrm{ng} / \mathrm{mL})$ were risk factors for carotid atherosclerosis in perimenopausal women $(\mathrm{P}<0.05)$, as shown in Table 3.

Table 3: Multivariate logistic regression analysis of carotid atherosclerosis in postmenopausal women. Multi-factor logistics regression analysis was used to analyze the correlation among indicators.

\begin{tabular}{|c|c|c|c|c|c|c|}
\hline Variable & B value & SE & Wals 2 & OR value & 95\%CI & P value \\
\hline $\mathrm{BMI}(\geq 25(\mathrm{~kg} / \mathrm{m} 2)$ & 0.538 & 0.249 & 4.668 & 1.713 & $1.051 \sim 2.790$ & $0.031 \star$ \\
\hline HBP & 0.589 & 0.257 & 5.252 & 1.802 & $1.089 \sim 2.982$ & $0.022 \star$ \\
\hline Diabetes & 0.602 & 0.243 & 6.137 & 1.826 & $1.134 \sim 2.940$ & $0.014 \star$ \\
\hline $\mathrm{TG}(\geq 1.7 \mathrm{mmol} / \mathrm{L})$ & 0.498 & 0.231 & 1.645 & 1.046 & $1.046 \sim 2.588$ & $0.032 \star$ \\
\hline Osteocalcin $(\leq 16.0 \mathrm{ng} / \mathrm{mL})$ & 0.559 & 0.239 & 5.471 & 1.749 & $1.095 \sim 2.794$ & $0.020 \star$ \\
\hline
\end{tabular}

\section{Discussion}

Perimenopausal period refers to women from sexual maturation period gradually into the transitional period of old age, namely from ovarian function decline to stop completely. The most prominent manifestation during this period is menopause. In 1994, the world health organization held a special meeting in Geneva recommended that "menopause" be renamed perimenopause and gave the definition of perimenopause refers to a period of time before menopause, the occurrence of endocrine, biological changes and clinical characteristics related to menopause to 12 months after menopause. Menopause is an inevitable physiological process in the life course of every woman. Menopause represents the decline of ovarian function, endocrine disorder and the termination of reproductive function. The age of menopause varies greatly among individuals,generally occurring between 45 and 55 years old. The average age of menopause for urban women in China is 49.5 years and that for rural women is 47.5 years. Menopause is divided into natural menopause and artificial menopause two kinds. Natural menopause refers to the loss of ovarian function and the permanent cessation of menstruation. Artificial menopause refers to the surgical removal of both ovaries (to preserve or remove the uterus) or other methods to stop ovarian function, such as chemotherapy or radiotherapy, to stop menstruation. According to the physiological process of change can be divided into: (1) menopause transition (menopausal transition) : refers to the menstrual cycle began to change to the front of the last menstrual periodof time. (2) the menopause (menopause) : due to the loss of ovarian function and make menstruation stop forever. Clinically, patients with amenorrhea for 12 months without significant pathological changes or other physiological reasons, will be considered menopause. (3) late menopause (postmenopause) : refers to after the last menstrual period. Perimenopausal period is the period from the flourishing period to the weak period of female fertility, the women in this stage are often accompanied by symptoms such as lumbar and knee tenderness, pain, etc., but 
also will show osteoporosis, increased bone brittleness, increased incidence of osteoporosis, seriously affecting the quality of life of perimenopausal women. In addition, perimenopausal period woman still is easy to produceobesity, dyslipidemia, hypertensive, coronary heart disease, arteriosclerosis et al cardiovascular and cerebrovascular disease. Atherosclerosis is a pathological process of arterial vascular wall thickening, hardening, and gradually decreasing vascular elasticity, which often occurs in the early stage of cardiovascular diseases. Patients show a systemic diffuse pathological state, and most of the involved systems are large and medium-sized arteries, which may cause damage to the functions of heart, kidney, brain and other organs [5]. The carotid artery is one of the most commonly involved sites of atherosclerosis. Some scholars show that the incidence of atherosclerosis in perimenopausal women is higher than that in male of the same age, which may be related to the dysfunction of autonomic nerve and the decline of nest-warming function in perimenopausal women. It has been pointed out that obesity, high TG, LDLc, low HDLc were risk factors for cardiovascular diseases, and the thickening of the vascular wall is also a marker of atherosclerosis [6].

The results of this study showed that 25 of the 89 perimenopausal women developed cervical atherosclerosis (the prevalence rate was $28.09 \%$ ). After analyzing the clinical data, it was found that the BMI and TG of perimenopausal women with cervical atherosclerosis were higher but the HDLc were lower than those without cervical atherosclerosis, indicating that the increase of BMI and dyslipidemia was related to carotid atherosclerosis in perimenopausal women. Obesity, Atherosclerosis and osteoporosis often coexist. The occurrence of atherosclerosis is related to genetic factors, glucose and lipid metabolism, hormone level, etc. The correlation between BMI \& atherosclerosis and bone metabolism level has been a research hotspot in recent years. Arterial calcification is also one of the pathological mechanisms of atherosclerosis. Obesity, arterial calcification were similar to bone formation. The essence of vascular calcification is the induction of inflammatory cytokines mainly come from adipo tissues at the vascular level into programmed osteogenesis, which mainly involves the activation of angiogenesis, the enhancement of bone conversion and the abnormal metabolism of minerals. This study found that the contents of HDLc, $25(\mathrm{OH}) \mathrm{D}_{3}$, CTX and osteocalcin in perimenopausal women with cervical atherosclerosis were lower than those in women without cervical atherosclerosis, while the contents of PTH increased significantly. In addition, correlation analysis showed that BMI, TG , HDLc, $25(\mathrm{OH}) \mathrm{D}_{3}$, CTX , osteocalcin and PTH levels were positively correlated with IMT,while age, TC, LDLc, and PINH levels were negatively correlated with IMT. PTH is parathyroid hormone, which can promote the release of bone calcium and phosphorus and other mechanisms to maintain the balance of blood calcium and phosphorus and increase the blood calcium content. Serum PTH content in patients with renal failure is related to the occurrence and progression of atherosclerosis, which may be a risk factor for atherosclerosis. Wang Cuixia et al. [7] found in their study on the correlation between bone metabolism index and atherosclerotic disease in elderly patients that serum PTH content in atherosclerotic patients increased significantly, while serum 25 -hydroxyvitamin D content decreased. $25(\mathrm{OH}) \mathrm{D}_{3}$ is a fat-soluble vitamin that is involved in bone metabolism and can inhibit calcification of sub-atherosclerotic plaques by regulating $\mathrm{Ca} 2+/ \mathrm{CaM}$ signaling [8]. Lower $25 \mathrm{OH}) \mathrm{D}_{3}$ content can affect the body of the intestinal calcium absorption, reduce the content of blood vessel, causing PTH levels increase [9]. CTX, a metabolite of collagen type I, is oneof the markers of bone resorption and bone transformation and can be used as a predictor of bone loss and new fractures in the elderly $[10,11]$. Osteocalcin is mainly synthesized by osteoblasts and plays a regulatory role in the activities of osteoclasts and precursor cells, reflecting the turning point of osteoclasts and osteoblasts [12]. Wu bingjie et al. [13] believed that decreased CTX content was an independent risk factor for coronary heart disease, and the degree of decreased CTX content was negatively correlated with the severity of coronary atherosclerosis in postmenopausal women. Deng jing et al. [14] found that carotid atherosclerosis in patients with type 2 diabetes is closely related to bone mineral density, and the intima media thickness of carotid artery is negatively correlated with the bone mineral density of lumbar spine 1-4, femoral neck and total hip. A similarcorrelation was found in this study, suggesting a correlation between glucolipid metabolism \& carotid atherosclerosis and abnormal bone metabolism in perimenopausal women. Through single-factor analysis and multi-factor logistic regression analysis on the clinical data of menopausal women with and without carotid atherosclerosis, the results showed that hypertension, diabetes, overweight or obesity, higher TG and lower osteocalcin were risk factors for cervical atherosclerosis in perimenopausal women. The mechanism may be related to factors such as high blood lipid level and high blood glucose level, which put the body in a state of oxidative stress and inflammatory response, increase the release of inflammatory factors in the body, and damage to vascular endothelial tissue, which is a stimulating factor for atherosclerosis and vascular calcification [15]. Decreased osteocalcin level is a risk factor for carotid atherosclerosis, possibly because osteocalcin can regulate the role of vasodilatory factors and inflammatory factors, inhibit vascular calcification, and thus affect the formation of atherosclerosis [16]. Osteocalcin can also affect glucose and lipid metabolism and hormone levels, thus indirectly affecting the process of atherosclerosis [17-19]. The limitation of this study is that the number of samples is not enough, so the lack of correlation between TC and LDLc and the formation of atherosclerotic plaques may be related to this. In addition, some patients with diabetes and hypertension with a long course of disease may also be affected by their clinical routine medication. Further expanding the sample size and conducting stratified analysis are expected to provide more accurate evidence-based medical evidence for their differences. 
In conclusion, overweight or obesity and the development of carotid atherosclerosis in perimenopausal women is closely related to abnormal bone metabolism [20-21], in which PTH level is positively correlated with the degree of carotid atherosclerosis, while $25(\mathrm{OH}) \mathrm{D}_{3}$, CTX and osteocalcin level are negatively correlated with the degree of carotid atherosclerosis, and hypertension, diabetes, abnormal lipid metabolism, obesity and decreased osteocalcin content are important risk factors for the occurrence of carotid atherosclerosis in perimenopausal women.

\section{Acknowledgement}

\section{Funding}

This study is supported by the Hong Kong General Research Fund. (201306151203), HKU Small Project Funding (201409176257), Science and technology program of medical and health of Shenzhen health committee of the NPC (SZFZ2017057), "Miao miao" cultivation program of Shenzhen hospital of southern medical university (2017MM01 and 2018MM11), Shenzhen Baoan district science and technology program basic research project (2017JD006 and 2017JD085).

\section{References}

1. Qing Ren, Xinyu Yu, Fujiu Liao, Xiaofan Chen, Dongmei Yan, et al. (2018) Effects of guasha therapy on perimenopausal syndrome: A systematic review and meta-analysis of randomized controlled trials. Complement Ther Clin Pract 31: 268-277.

2. Thurston RC, Chang Y, Mitchell EB, Jennings JR, Ka nel R, et al. (2018) Physiologically assessed hot flashes and endothelial function among midlife women. Menopause 25(11): 1354-1361.

3. Zhaleh SS, Rezaie EH, NasrinMilani, Rezaie FE, Rezaie AE (2018) Evaluation of bone mineral density in perimenopausal period. Arch Bone Jt Surg 6(1): 57-62.

4. Sonographer Branch of Chinese Physicians Association (2009) Guidelines for vascular ultrasound examination.Chinese Journal of Ultrasound Imaging 18(10): 911-920.

5. Mozaffarian D, Benjamin EJ, Go AS, Arnett DK, Blaha MJ, et al. (2016) Heart disease and stroke statistics-2016 update: A report from the American Heart Association. Circulation 133(4): 338-360.

6. Olszanecka A, Dragan A, Jaszcz KK, Czarnecka D (2014) Influence of metabolic syndrome and its components on subclinical organ damage in hypertensive perimenopausal women. Adv Med Sci 59(2): 232-239.

7. Mueller PA, Zhu L, Tavori H, Huynh K, GiunzioniI, et al. (2018) Deletion of macrophage low-density lipoprotein receptor-related protein 1 (LRP1) accelerates atherosclerosis regression and increases c-c chemokine receptor type 7 (CCR7) expression in plaque macrophages . Circulation 138(17): 1850-1863.

8. Ping Y, Xinggang C, Bo L (2008) 1,25-(OH)_2D_3 regulates endothelial autophagy and inhibits atherosclerotic calcification through $\mathrm{Ca} \sim(2+) /$ CaM signaling.Chinese Journal of Arteriosclerosis 26(11): 1091-1098.

9. Pingda B, Zhangxuan S, Xiuyang Li(2008) Older men serum collagen type C-peptide cross-linked at the end of the independent factors.Chinese Journal of Osteoporosis and Bone Mineral Disease 11(6): 564-569.

10. Bian P, Li X, Ying Q Chen J, Jin X, et al. (2015) Factors associated with low femoral neck bone mineral density in very elderly Chinese males. Arch Gerontol Geriatr 61(3): 484-488.

11. Dai Z, Wang R, Ang LW, Yuan JM, Koh WP (2016)Bone turnover biomarkers and risk of osteoporotic hip fracture in an Asian population. Bone 83: 171-177.

12. Tacey A, Qaradakhi T, Speranza TB, Hayes A, Zulli A, et al. (2018) Potential role for osteocalcin in the development of atherosclerosis and blood vessel disease. Nutrients 10(10).

13. Yan Ling, Zhen Wang, Bingjie Wu, Xin Gao (2018) Association of bone metabolism markers with coronary atherosclerosis and coronary artery disease in postmenopausal women. J Bone Miner Metab 36(3): 352-363.

14. Freedman BI, Divers J, Russell GB, Palmer ND, Wagenknecht LE, et al. (2015) Vitamin D associations with renal, bone, and cardiovascular phenotypes: African American-Diabetes Heart Study. J Clin Endocrinol Metab 100(10): 3693-3701.

15. Kang JH (2016) Association of serum osteocalcin with insulin resistance and coronary atherosclerosis. J Bone Metab 23(4): 183-190.

16. Yang SW, Hennessy RR, Khosla S, Lennon R, Loeffler D, et al. (2017) Circulating osteogenic endothelial progenitor cell counts: new biomarker for the severity of coronary artery disease. Int J Cardiol 227: 833-839.

17. Guedes JAC, Esteves JV, Morais MR,Zorn TM, Furuya DT (2018) Osteocalcin improves insulin resistance and inflammation in obes mice: Participation of white adipose tissue and bone. Bone 115: 68-82.

18. Goetz LG, Mamillapalli R, Sahin C, Zolbin MM, Ge G, et al. (2017) Addition of estradiol to cross-sex testosterone therapy reduces atherosclerosis plaque formation in female ApoE-/-mice. Endocrinology 159(2): 754762.

19. Lim Y, Chun S, Lee J H, Baek KH, Lee WK, et al. (2016) Association of bone mineral density and diabetic retinopathy in diabetic subjects: the 2008-2011 Korea National Health and Nutrition Examination Survey. Osteoporos Int 27(7): 2249-2257.

20. Tongdee P, Loyd RA, Kanoksin S, KanjanawetangJ, Winwan K, et al. (2016) Clinical usefulness of lipid ratios to identify subclinical atherosclerosis in perimenopausal/menopausal women. J Med Assoc Thai 99(7): S36-S41.

21. El Khoudary SR, Ceponiene I, Samargandy S, Stein JH, Li D, et al. (2018) HDL (high-density lipoprotein) metrics and atherosclerotic risk in women. Arterioscler Thromb Vasc Biol 38(9): 2236-2244. 